\title{
High-speed analysis of nuclear emulsion films with the use of dry objective lenses
}

\author{
I. Kreslo, ${ }^{a *}$ M. Cozzi, ${ }^{c}$ A. Ereditato, ${ }^{a}$ M. Hess, ${ }^{a}$ J. Knuesel,,${ }^{a}$ I. Laktineh, ${ }^{b}$ M. Messina, ${ }^{a}$ \\ U. Moser, ${ }^{a}$ C. Pistillo, ${ }^{a}$ K. Pretzl, ${ }^{a}$ L. Scotto Lavina, ${ }^{a}$ G. Sirri, ${ }^{c}$ H.-U. Schutz ${ }^{a}$ and \\ V. Tioukov ${ }^{d}$ \\ ${ }^{a}$ Laboratory for High Energy Physics, University of Bern, Bern, Switzerland \\ ${ }^{b}$ IPNL, IN2P3-CNRS and Université C. Bernard Lyon I, Lyon, Villeurbanne - France \\ ${ }^{c}$ Dip. di Fisica dell'Università di Bologna and INFN, Bologna, Italy \\ ${ }^{d}$ Dip. di Fisica dell'Università di Napoli and INFN, Napoli, Italy \\ E-mail: Igor.Kreslo@cern.ch
}

\begin{abstract}
The extensive use of nuclear emulsions as precise tracking detectors in experimental physics has been made possible due to recent advances in the production of novel emulsion films and to the development of automatic scanning devices. The scanning speed of such systems has exceeded the level of $20 \mathrm{~cm}^{2}$ of emulsion surface per hour. High-speed automatic scanning systems, such as those developed by the OPERA Collaboration, are able to reconstruct particle tracks in nuclear emulsions with excellent accuracy. However, the high-magnification oil immersion objectives used in these systems assume deposition and removal of oil onto and from the emulsion films. This is a major technological obstacle in the automatization of the emulsion feeding to the microscope, as required for large scale use as in the case of the OPERA neutrino oscillation experiment. In order to overcome this problem, an innovative technique of nuclear emulsion films scanning with the use of dry objective lenses has been developed and successfully applied to the experiment.
\end{abstract}

KEYWORDS: Hybrid detectors; Particle tracking detectors; Large detector systems for particle and astroparticle physics; Particle detectors.

${ }^{*}$ Corresponding author. 


\section{Contents}

1. Introduction 1

2. Basic parameters in optical microscopy 2

3. Scanning performance of the OPERA European Scanning System with oil immersion objectives

4. Performance of dry objectives with the OPERA ESS

5. Conclusions

\section{Introduction}

The history of nuclear emulsions usage for the detection of charged particles falls back to the fifties of the 20th century. The method is based on the fact that ionizing particles passing through a gelatin layer saturated by silver halide leave so-called activation centers, just like photons in conventional photography [1], 2]. The first detectors based on this principle were made of glass plates with a thin sensitive layer deposited onto the surface. With the time, more and more polymer materials came to the market, so glass was replaced by cellulose-based compounds like cellulose tri- and diacetate, and nuclear emulsions were successfully applied to various particle physics experiments (see e.g. [3]-17]).

The extensive use of nuclear emulsions as precise tracking detectors in experimental physics has been made possible due to recent advances in the production of novel nuclear emulsion films and to the development of new generation automatic scanning devices. Emulsion films with dimensions of $12.5 \times 10 \mathrm{~cm}^{2}$ have been commercially produced by the Fuji Film ${ }^{1}$ company for the CERN-LNGS OPERA neutrino oscillation experiment ${ }^{2}[19]$.

In order to reconstruct particle tracks recorded in the emulsion films, they must be developed and then scanned with optical microscopes, given that the silver grains constituting the particle tracks have micrometric dimensions. In the old days emulsion scanning was performed by human operators by means of manual microscopes. This technique (so-called "eye scan") required large manpower, and operators had to be highly trained and qualified in order to achieve good track recognition efficiency. As the scale of experiments has grown, the necessity of computer controlled automatic procedure became mandatory. In such systems a computer controls the movements of object table and microscope head and performs the digital image acquisition and processing.

\footnotetext{
${ }^{1}$ Fuji Film, Minamiashigara, 250-0193, Japan

${ }^{2} \mathrm{An}$ appearance experiment to search for $v_{\mu}-v_{\tau}$ oscillations in the CNGS beam [18].
} 
An important step in this direction was made at the Nagoya University under the leadership of K. Niwa. The system called Track Selector was developed in 1982 and successfully used in the CHORUS [16] and DONUT [17] experiments. It evolved its scanning ability from 0.2 microscope views per second in 1982 to 30 views per second in 2001 [20, 21].

A similar progress has recently been made in Europe following earlier developments [22]. A system able to scan up to $20 \mathrm{~cm}^{2} /$ hour of emulsion surface with real-time track reconstruction, the so-called European Scanning System (ESS), has been developed by the european groups of the OPERA collaboration [23, 24].

Historically, in order to obtain high spatial resolution objective lenses with high numerical aperture were used. Up to recent times such lenses were only of the immersion type. Recent developments by leading microscopy companies like Nikon, ${ }^{3}$ Olympus, ${ }^{4}$ Zeiss ${ }^{5}$ and others resulted in new types of dry objective lenses. Such lenses allow today obtaining as high performance as the immersion ones, but they are easier to use mostly in relation to recent experimental largescale applications. In this paper the performance of a high-resolution automatic scanning system employing dry objective lenses is presented.

\section{Basic parameters in optical microscopy}

An optical microscope is characterised by a set of parameters that define its performance. The most important of them are listed below in order to ease the understanding of the experimental results presented in this paper.

- Numerical aperture.

The numerical aperture $(N A)$ of the objective is defined as

$$
N A=n \cdot \sin (A / 2)
$$

where $n$ is the refraction index of the medium between the objective lens and the specimen, and $\mathrm{A}$ is the angular aperture, that is the maximum opening angle of the light cone that the lens can see. For infinity-corrected objective lenses this angle is $2 \cdot \arctan (R / F)$, where $R$ is the radius of the front lens and $F$ is the focal distance. The numerical aperture defines a number of important parameters, such as lateral resolution, field depth, optical efficiency, etc. In fact, in order to derive these parameters the only knowledge of the $N A$ of the objective lens is not sufficient. The light cone arriving at the objective can be limited by the illumination system. In this case the maximum $N A$ of the objective is not meaningful. Therefore, in the following discussion we will use an effective numerical aperture $N A_{\text {eff }}$, calculated separately for each particular case.

- Lateral spatial resolution.

\footnotetext{
${ }^{3}$ Nikon Instruments Inc. 1300 Walt Whitman Road, Melville, NY 11747-3064, U.S.A.

${ }^{4}$ Olympus America Inc. 3500 Corporate Parkway, P.O. Box 610, Center Valley, PA 18034-0610

${ }^{5}$ Carl Zeiss MicroImaging, Inc., One Zeiss Drive Thornwood, NY 10594
} 


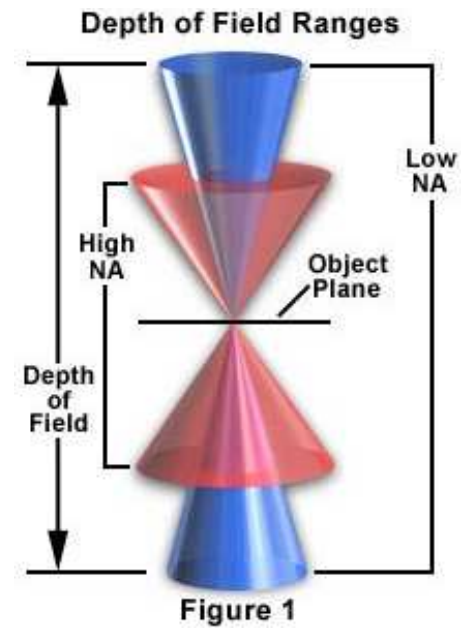

Figure 1. Field depth depends on the objective lens numerical aperture.

The lateral resolution of a high magnification microscope system with a digital camera has two main contributions: the camera pixel size and the diffraction limit. The latter is defined as

$$
R=\frac{0.66 \lambda_{0}}{N A_{\mathrm{eff}}}
$$

where $\lambda_{0}$ is the wavelength of the illumination light and $N A_{\text {eff }}$ is the effective numerical aperture of the system.

- Longitudinal resolution: depth of field.

The depth of field $(d)$ is defined as the distance between the nearest and the furthest objects in focus (see figure 11] [25]. A commonly used formula for the depth of field gives:

$$
d=\frac{\lambda_{0} \cdot n}{\left(N A_{\mathrm{eff}}\right)^{2}}+\frac{n \cdot e}{M \cdot N A_{\mathrm{eff}}}
$$

where $\lambda_{0}$ is the wavelength of the illumination light, $n$ is the refraction index of the medium between the objective lens and the emulsion film, $N A_{\text {eff }}$ is the effective numerical aperture of the system, $e$ is the spatial resolution of the readout system (in our case the CMOS sensor pixel size) and $M$ is the objective magnification. The dependence of the field depth on the numerical aperture is shown in figure 2, where four points corresponding to four different objectives produced by NIKON are indicated. Here we assume that the illumination system provides the necessary light cone such that it does not limit the nominal numerical aperture of the objectives. Later on we will show that this is not always the case.

\section{Scanning performance of the OPERA European Scanning System with oil immer- sion objectives}

Nuclear emulsion is composed by a gelatin layer saturated by a micro-crystallic silver halide, usually $\mathrm{AgBr}$. A charged particle passing through the emulsion leaves the so-called activation centers 


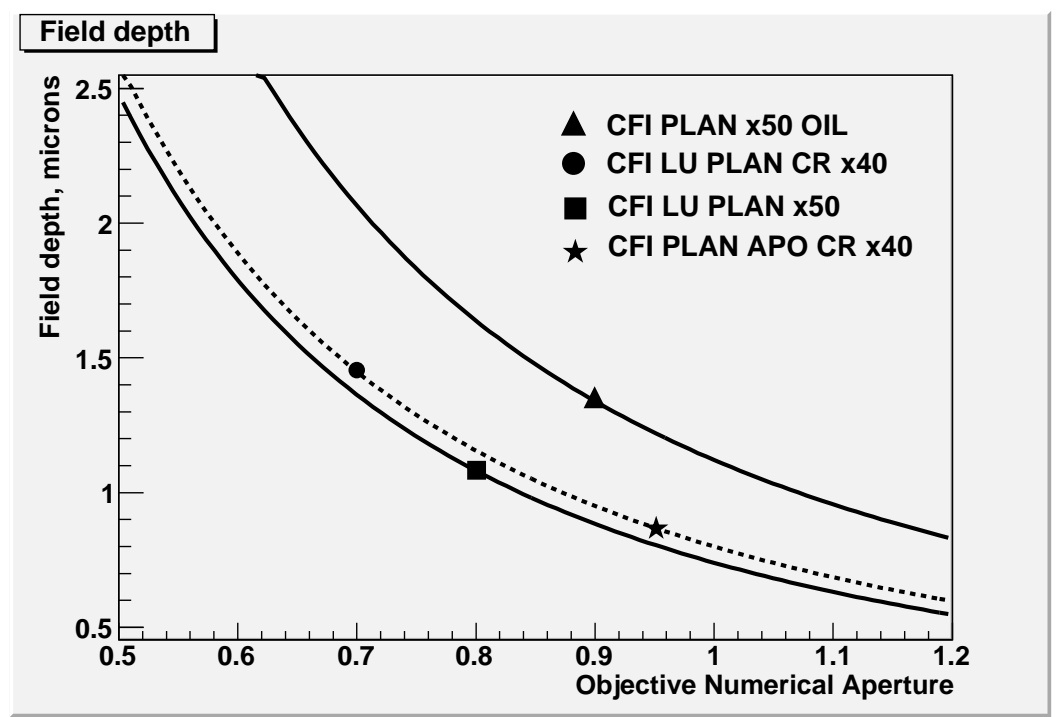

Figure 2. Field depth versus objective numerical aperture. Assumptions: camera pixel size of $12 \times 12 \mu \mathrm{m}^{2}$, refraction indices for air $n_{\text {air }}=1.0$, for immersion oil $n_{\text {oil }}=1.515$. Top curve: immersion objective; bottom curves: dry objective. Solid lines: objective magnification $M=50$; dashed line $M=40$. The points correspond to four NIKON objectives used in the emulsion scanning.

at the surface of the micro-crystals. During the emulsion development the silver grains start to grow from such activation centers and fill the whole crystals. The number of such grains is related to the ionizing properties of the particle. For relativistic particles (also called Minimum Ionizing Particles, or MIP) the grain number equals approximately to 30 grains per $100 \mu \mathrm{m}$ of particle track. The size of the grains is primarily defined by the size of $\mathrm{AgBr}$ micro-crystals. It is also affected by the emulsion development regime (temperature, solution content, etc.).

The emulsion films produced commercially by Fuji Film for the OPERA neutrino oscillation experiment are made of two sensitive emulsion layers deposited on both sides of a cellulose triacetate film. The thickness of this film, also called base, is $200 \mu \mathrm{m}$. The thickness of the sensitive emulsion layers is $50 \mu \mathrm{m}$ (see figure 3). The production technology is such that each sensitive layer is made in two passes. In each pass $24 \mu \mathrm{m}$ thick sensitive layer is deposited together with $1 \mu \mathrm{m}$ thick insensitive protection layer on top. Thus the surface of the sensitive layer is protected from mechanical and chemical damage. The side effect is the presence of the insensitive $1 \mu \mathrm{m}$ thick layer in the middle of the sensitive layer. This approach results in a high uniformity of the layer thickness (better than $1 \mu \mathrm{m}$ ).

Minimum ionizing particles leave about 15 grains in the depth of the emulsion layer for normal incidence. After development the emulsion thickness is reduced to approximately $45 \mu \mathrm{m}$. In order to detect a particle track, the microscope takes a number of images focused at different depth of the emulsion layer. Each of these images represents a tomographic cut of the emulsion, assuming that the focal depth is comparable to the distance between consecutive images (see figure $\rrbracket$ ). By combining a "pile" of images the particle track can be reconstructed by associating grains in different images with the aid of suitable computer algorithms. The distance between images must be small enough to avoid missing silver grains in between. The track reconstruction time rapidly increases 

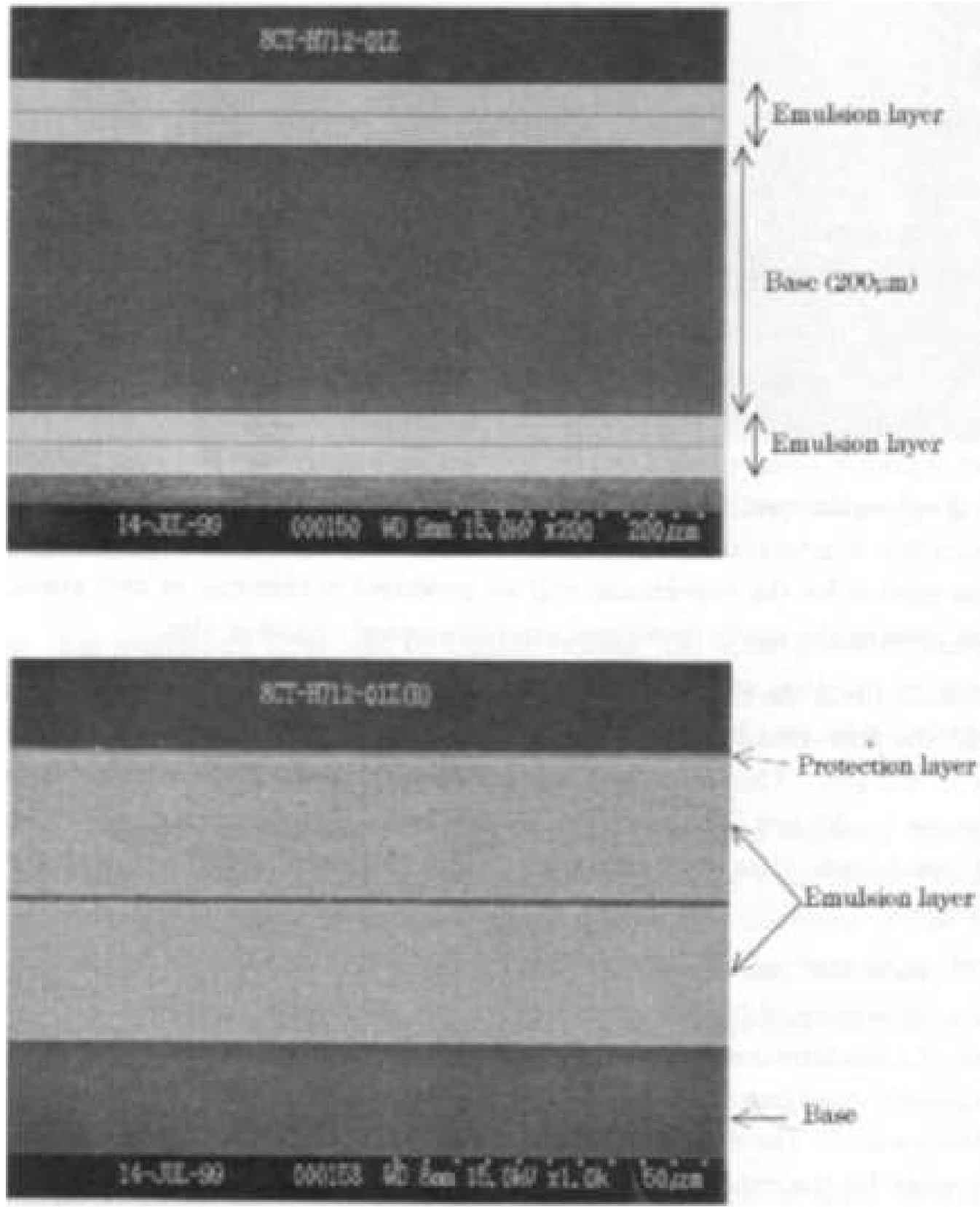

Figure 3. The transverse structure of the nuclear emulsion film, used in OPERA experiment. On the lower photo the detailed structure of one emulsion layer is shown. The insensitive gelatin layer of 1 micron thickness in the middle is well visible.

with the number of images. For the OPERA scanning microscopes (ESS) 15 images are taken as a compromise. This results in an image-to-image distance of about $3 \mu \mathrm{m}$.

A detailed description of the OPERA European Scanning System with oil immersion objective lenses is given in [23, 24]. The optical scheme of this system is schematically shown in figure 5. A NIKON Achromat condenser with maximum numerical aperture of $N A_{\text {cond }}=0.8$ and working distance of $4.3 \mathrm{~mm}$ is utilized in this system. The condenser provides this aperture for air as the 


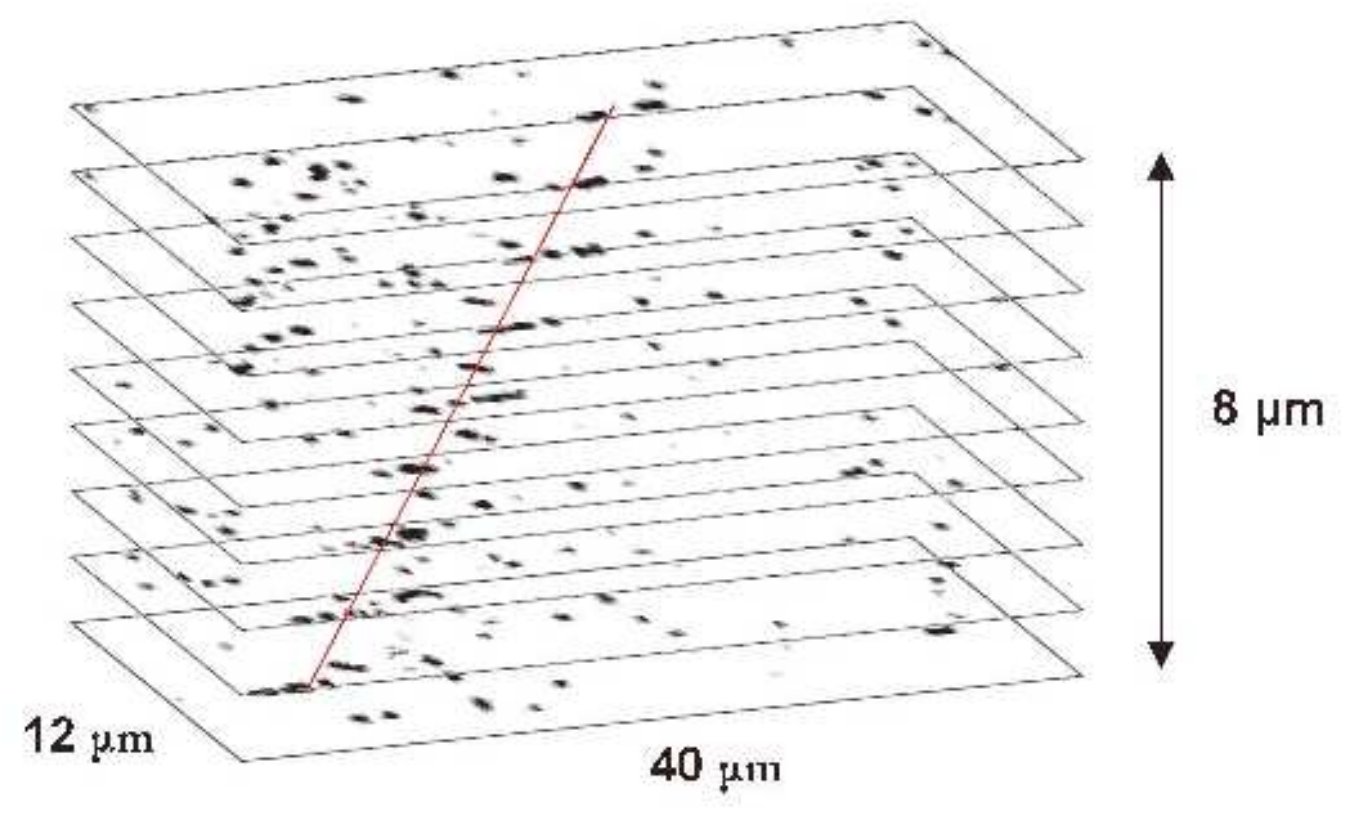

Figure 4. The combitation of 9 consecutive microscope images reveals the presence of a track. Small $12 \times 40 \mu \mathrm{m}$ fragment of a microscope view is shown.

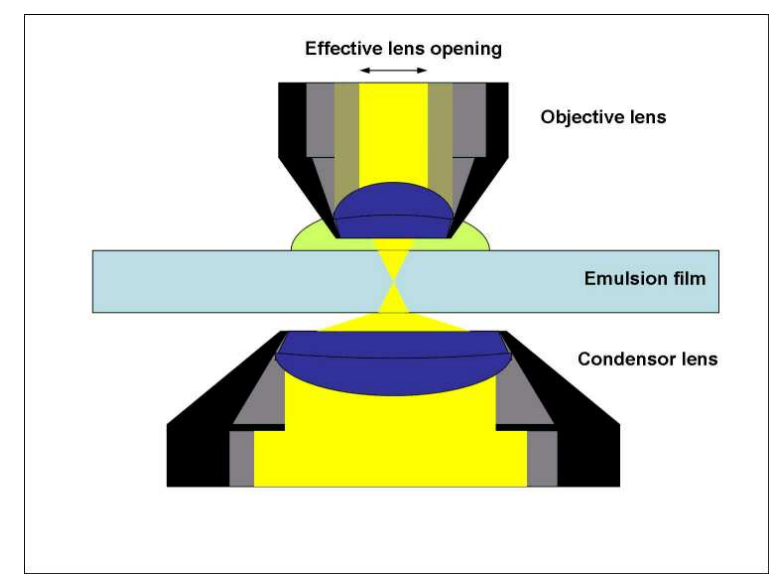

Figure 5. The optical scheme of the emulsion scanning microscope with immersion objective and light path in it. In this arrangement the effective numerical aperture is limited by the condenser lens.

medium between condenser lens and specimen. A NIKON CFI OIL Plan Achromat is used for the objective lens (in the following - immersion objective). The magnification of the objective is $\times 50$ and its numerical aperture $N A_{\text {obj }}=0.9$.

As seen in figure 5, the effective numerical aperture of the system is limited by the aperture of the condenser: $N A_{\text {eff }}=N A_{\text {cond }}=0.8$. The immersion oil has a refraction index $n_{\text {oil }}=1.515$ which matches to the refraction index of the emulsion. According to 2.2 and 2.3, the lateral and longitudinal resolutions of the system are $R_{\mathrm{oil}}=0.43 \mu \mathrm{m}$ and $d_{\mathrm{oil}}=1.23 \mu \mathrm{m}+0.45 \mu \mathrm{m}=1.68 \mu \mathrm{m}$.

The silver grains in the OPERA emulsion films have an average diameter of about $1 \mu \mathrm{m}[18]$ ]. A sophisticated image processing is applied in order to separate and select grain images from 


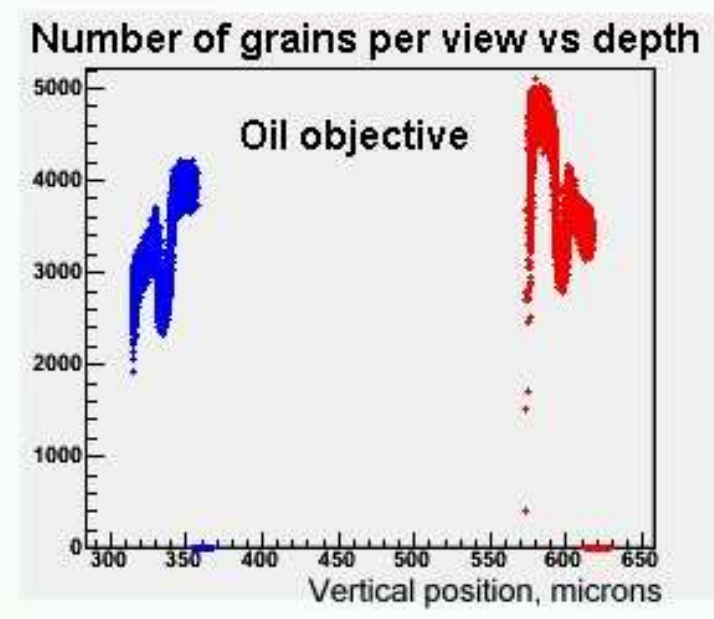

Figure 6. Number of grains per microscope view versus emulsion depth. Blue points are for the bottom and red points are for the top emulsion layers, respectively. Binarization thresholds are the same for both the emulsion layers, taken with oil immersion objective lens NIKON Plan Oil, $\times 50, N . A .=0.9$.
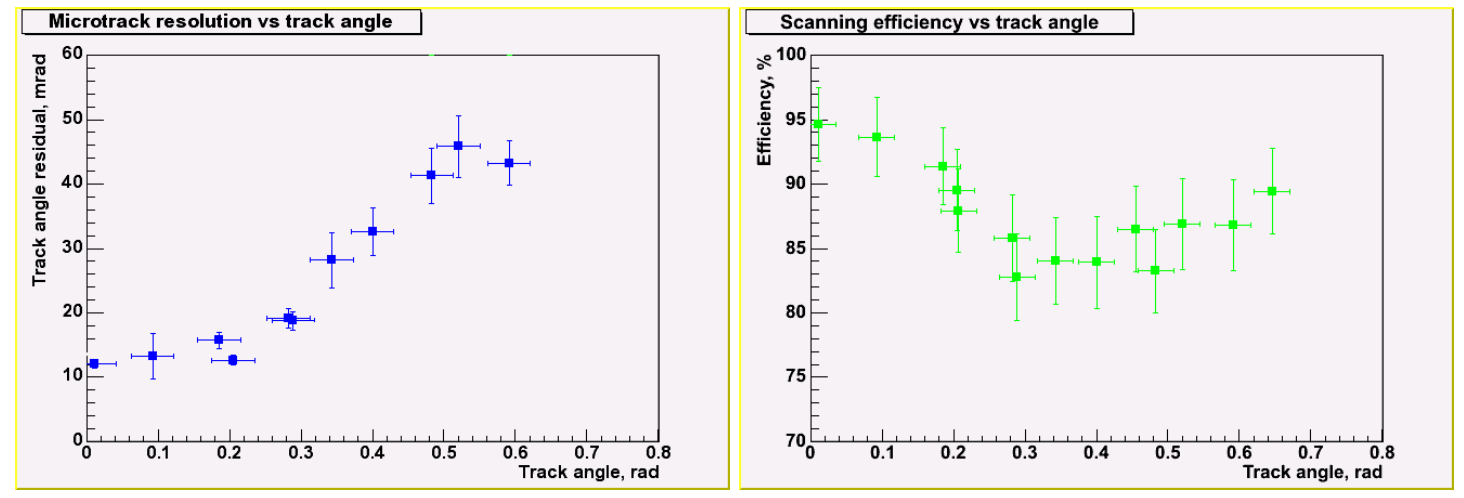

Figure 7. Dependence of the angular accuracy (left) and track reconstruction efficiency (right) on the track angle for oil immersion objective lens.

noise [23]. At the final step of the processing the image is binarized with a threshold. One grain is detected as such along some distance from its real position. This distance is defined by the transversal and longitudinal resolutions of the microscope (focal depth) as well as by the binarization threshold.

In figure 6 we show the number of detected silver grains per microscope view plotted against the vertical position of the focal plane inside the emulsion layer. The unit on the abscissa is $\mu \mathrm{m}$. The origin is set to approximately $300 \mu \mathrm{m}$ below the emulsion layer. The two emulsion layers are visible and in each layer the $1 \mu \mathrm{m}$ thick separation insensitive gelatin layer is well visible as a $20 \%$ drop in the number of grains.

The overall track reconstruction efficiency and accuracy are measured by the method described in [23] and are shown in figure 7. 


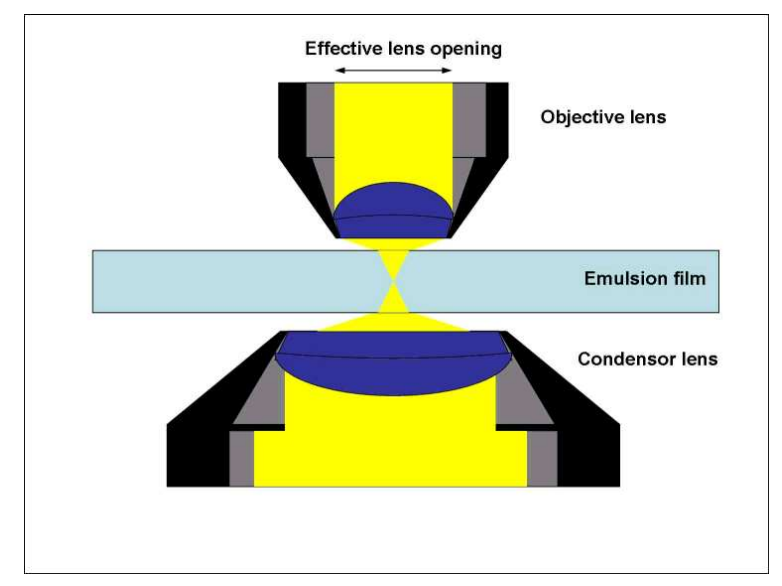

Figure 8. The optical scheme of the emulsion scanning microscope with dry objective. The light path is also shown.

\section{Performance of dry objectives with the OPERA ESS}

The optical scheme of the system that we developed for the use of dry objective lenses is schematically shown in figure 8. A NIKON Achromat condenser is used as in the case of immersion objective lenses. A NIKON CFI LU PLAN Achromat is used as microscope objective lens. The magnification of this objective is $\times 50$ and its numerical aperture $N A_{\mathrm{obj}}=0.8$. The effective numerical aperture of the system is $N A_{\text {eff }}=N A_{\text {cond }}=N A_{\text {obj }}=0.8$. According to 2.2 and 2.3 the lateral and the longitudinal resolutions for the system are $R_{\mathrm{dry}}=0.43 \mu \mathrm{m}$ and $d_{\mathrm{dry}}=0.81 \mu \mathrm{m}+0.3 \mu \mathrm{m}=1.11 \mu \mathrm{m}$.

From figure 8 one can see that the focal depth is $d=1.11 \mu \mathrm{m}$ only if the specimen (the silver grain) is located in the air (refraction index $n_{\text {air }}=1.0$ ). For grains in the depth of the emulsion layer space it will be shrinked according to the emulsion refraction index $n_{\mathrm{em}}=1.515$, so that the effective focal depth for the grains inside the emulsion layer becomes $d_{\mathrm{dry}}^{\mathrm{eff}}=1.515 \cdot 1.11 \mu \mathrm{m}=1.68 \mu \mathrm{m}$.

Compared to $R_{\text {oil }}=0.43 \mu \mathrm{m}$ and $d_{\text {oil }}=1.68 \mu \mathrm{m}$ one can see that replacing the immersion objective NIKON CFI OIL Plan Achromat by the dry objective NIKON CFI LU Plan Achromat does not change the principal parameters of the optical system, if the condenser lens with $N A_{\text {cond }}=$ 0.8 is used.

However, observing the emulsion image with a dry objective, one may notice a different image quality for the top and the bottom layers of the emulsion, that are separated from each other by 200 $\mu \mathrm{m}$ of plastic base. The presence of this relatively thick film with refraction index $n_{\mathrm{em}}=1.5$ in the optical path results in a slight decrease of the resolution due to spherical aberrations. This effect is difficult to quantify. On the left plot of figure 9 the number of detected silver grains per microscope view is shown, plotted against the vertical position of the focal plane inside the emulsion layer. The unit on the abscissa is $\mu \mathrm{m}$, and the origin is set to approximately $570 \mu \mathrm{m}$ below the emulsion layer. The decrease in vertical resolution is well visible from the figure for the bottom layer of the emulsion film (on the left in the picture). Also noticeable is the increase of field depth for the bottom layer.

The decrease of the grain size visible in the bottom layer can be compensated by lowering the binarization threshold (see right plot in figure 9). However, the larger field depth is not compensated 

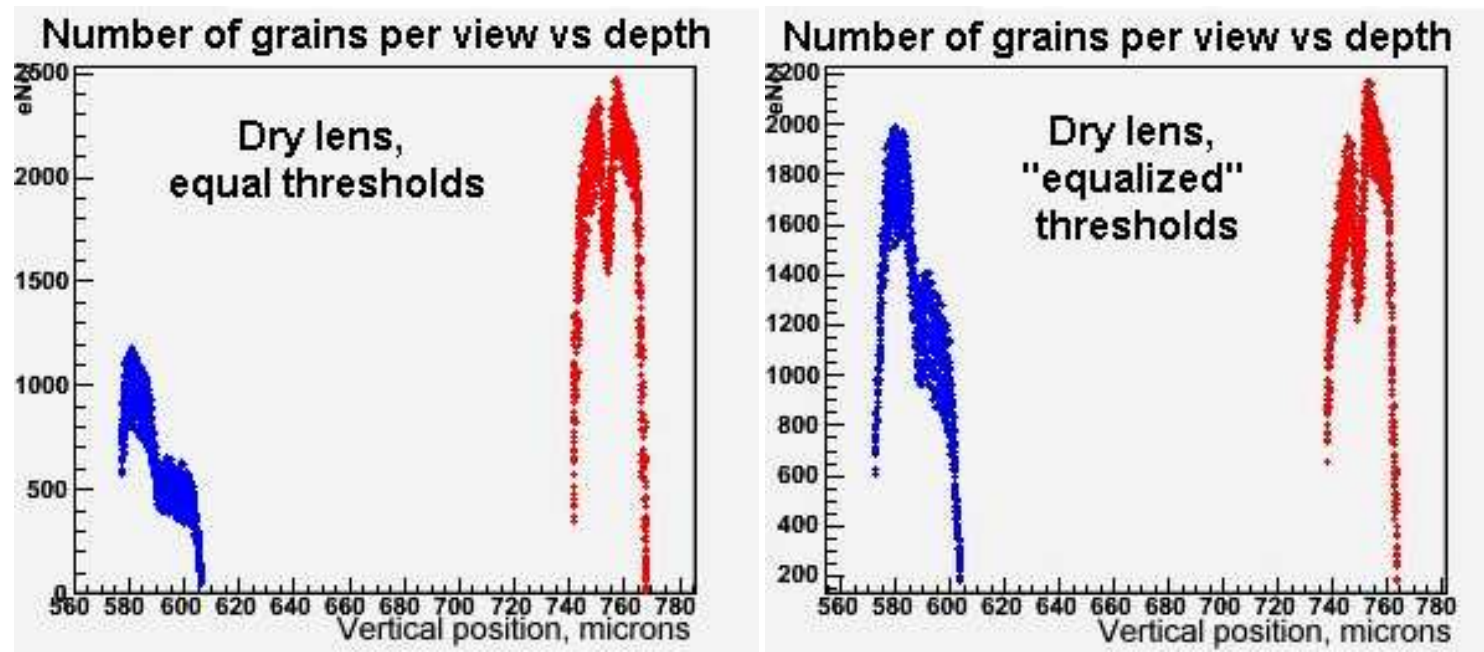

Figure 9. Number of grains per microscope view versus emulsion depth taken with NIKON Lu Plan dry, $\times 50$, N.A. $=0.8$. Binarization thresholds are the same (left plot) and adjusted to have the same number of grains for both emulsion layers (right plot).

by this approach. This may introduce as a side effect an increased number of fake tracks, especially at small angles, due to possible fog grain-to-grain association.

The best solution is to use the objective with a built-in correction system, which allows to optimize the resolution for a given thickness of material above the focal plane (the so-called cover glass correction). One of the objectives satisfying the above requirement is the NIKON PLAN Apochromat with $\times 40$ magnification, numerical aperture 0.95 and a range of cover glass thickness correction of $0.11-0.23 \mathrm{~mm}$. The thickness of the cover glass, for which the objective is optimized, can be set by the collar on the objective body (see figure 10). Similar results are shown for correction depth set to $0.09 \mathrm{~mm}, 0.14 \mathrm{~mm}$ and $0.23 \mathrm{~mm}$, respectively (figure 11). In the first plot of figure 11 the same situation as with the NIKON Lu Plan $\times 50$ objective lens is shown, namely the top layer is seen with very good resolution while the bottom one with a little worse resolution. In the last plot the situation is reversed. The objective is tuned to better see the bottom layer while the quality of the top layer image suffers in this case. However, a compromise can be found with intermediate setting (second plot), that shows excellent resolution for both top and bottom layers, resulting in $d_{\mathrm{dry}}^{\mathrm{eff}} \approx 1.7 \mu \mathrm{m}$.

The measurement of the track reconstruction efficiency and accuracy is made in the same way as for oil immersion objectives. The results are shown in the figure 12 .

In order to keep the thickness close to the original one after development the emulsion layer must contain a significant amount of water in the gelatin. Therefore, the humidity of the atmosphere around the emulsion must also be maintained at the level of $60-70 \%$. The illumination system of the microscope radiates rather large amount of light as well as heat, so it may heat up the emulsion layer and evaporate some water from it. This water will then be deposited as dew at the front lens of the dry objective, hence reducing the image quality. In order to avoid this, the objective must be kept at a temperature slightly higher than room temperature. A miniature heater made of a high resistivity wire is wound on the objective body, to serve for this purpose. The resistance of the heater is $50 \mathrm{Ohm}$ and the applied voltage is $12 \mathrm{~V} \mathrm{DC}$. The resulting temperature of the objective is 


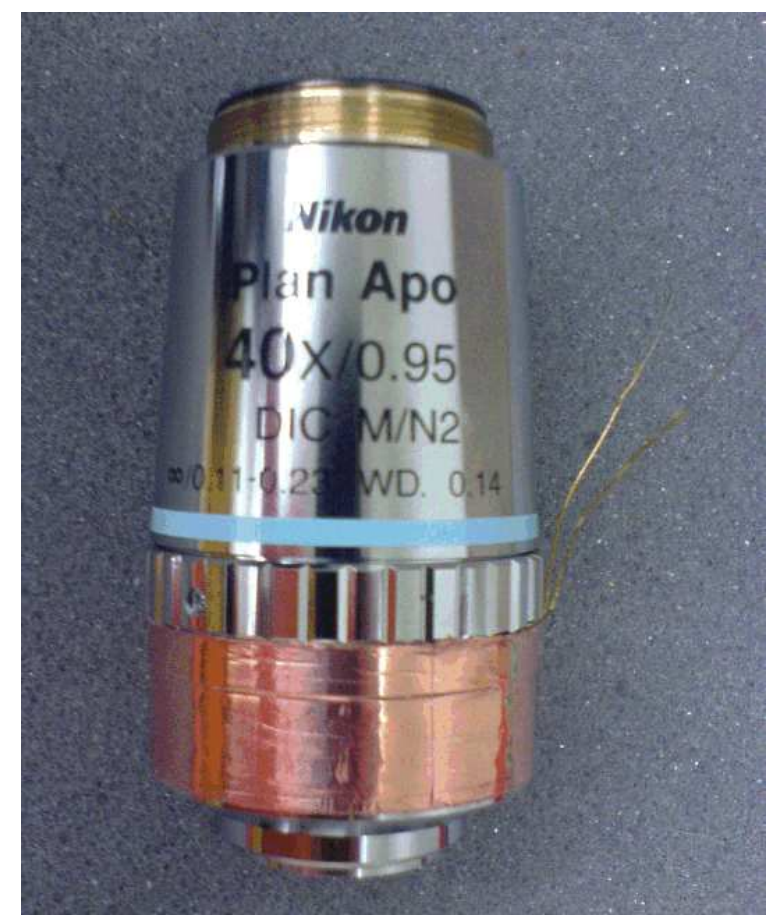

Figure 10. NIKON Plan Apochromat dry objective lens, $\times 40$ magnification, $N . A .=0.95$, with cover glass correction collar and heating coil.

about $35-40{ }^{\circ} \mathrm{C}$. This is enough to eliminate the risk of dew formation on the front lens.

\section{Conclusions}

The feasibility of using newly developed dry objective lenses with high numerical aperture for high-speed analysis of nuclear emulsion films is demonstrated with the application to the European Scanning System for the OPERA emulsion detector. The lateral resolutions of the ESS microscopes based on dry and oil immersion objective lenses are $R_{\mathrm{dry}}=R_{\mathrm{oil}}=0.43 \mu \mathrm{m}$. The longitudinal resolution with dry objective lens is $d_{\mathrm{dry}}=1.11 \mu \mathrm{m}$, that is noticeably better than with oil immersion objectives $d_{\mathrm{oil}}=1.68 \mu \mathrm{m}$. For reconstructed particle tracks, angular accuracy and track reconstruction efficiencies measured by means of the methods described in [23] are identical within the statistical errors for ESS equipped with dry objective lenses (figure 12) and oil immersion lenses (figure 7 ).

The use of dry objective lenses allows to avoid a number of difficulties connected to the use of the immersion oil, such as difficulties in the automatic placement of the emulsion films onto the microscope stage, storage problems for used emulsion films, potential health hazards from commonly used immersion oils, etc.

The Bern LHEP laboratory has been the first emulsion scanning laboratory utilizing five automatic microscopes with dry objectives and automatic emulsion plate changers [27] for high-speed analysis of OPERA emulsion films. The effectivness of our choice has been demonstrated by the ability in routine scan of films with high efficiency. As an example, an OPERA neutrino interaction event located in Bern with dry objective lenses and automatic film changer is shown in figure 13 . 

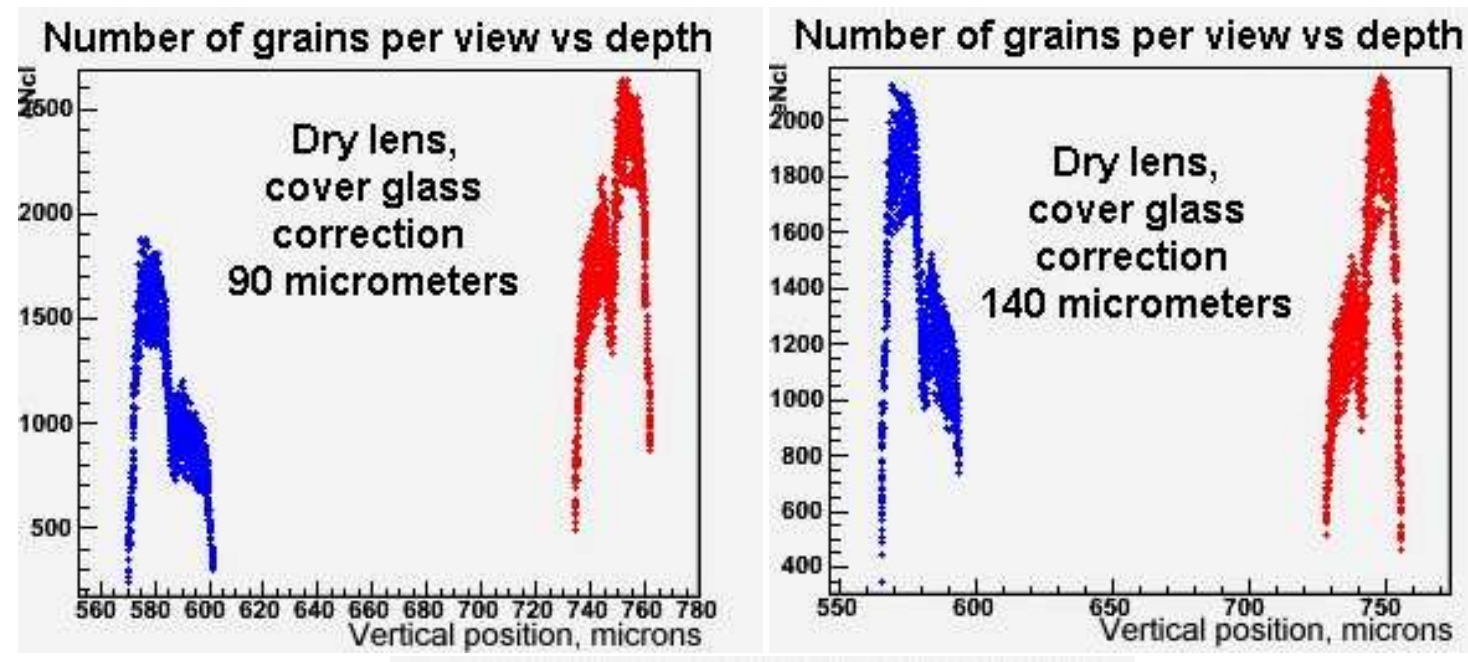

Number of grains per view vs depth

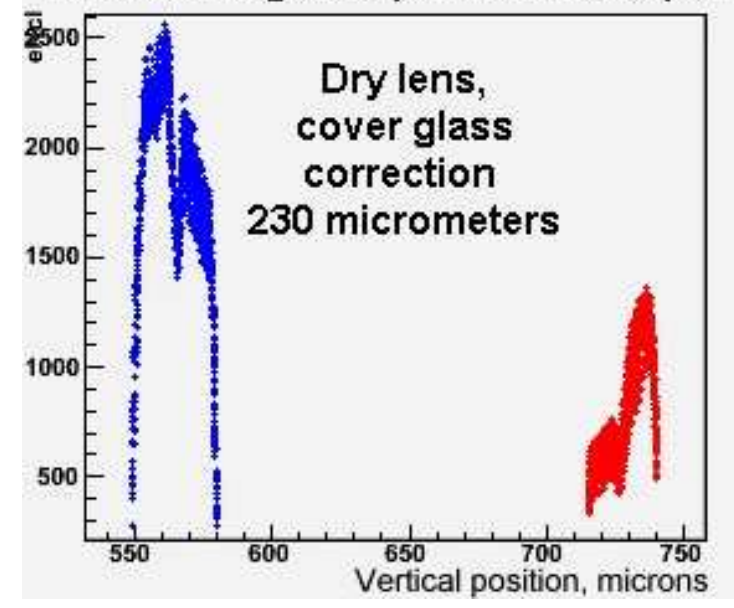

Figure 11. Number of grains per microscope view versus emulsion depth for the same binarization thresholds taken with NIKON Plan Apochromat dry objective, $\times 40$ magnification, N.A. $=0.95$, correction is set to $90 \mu \mathrm{m}$ (left top), $140 \mu \mathrm{m}$ (right top) and $230 \mu \mathrm{m}$ (left bottom)
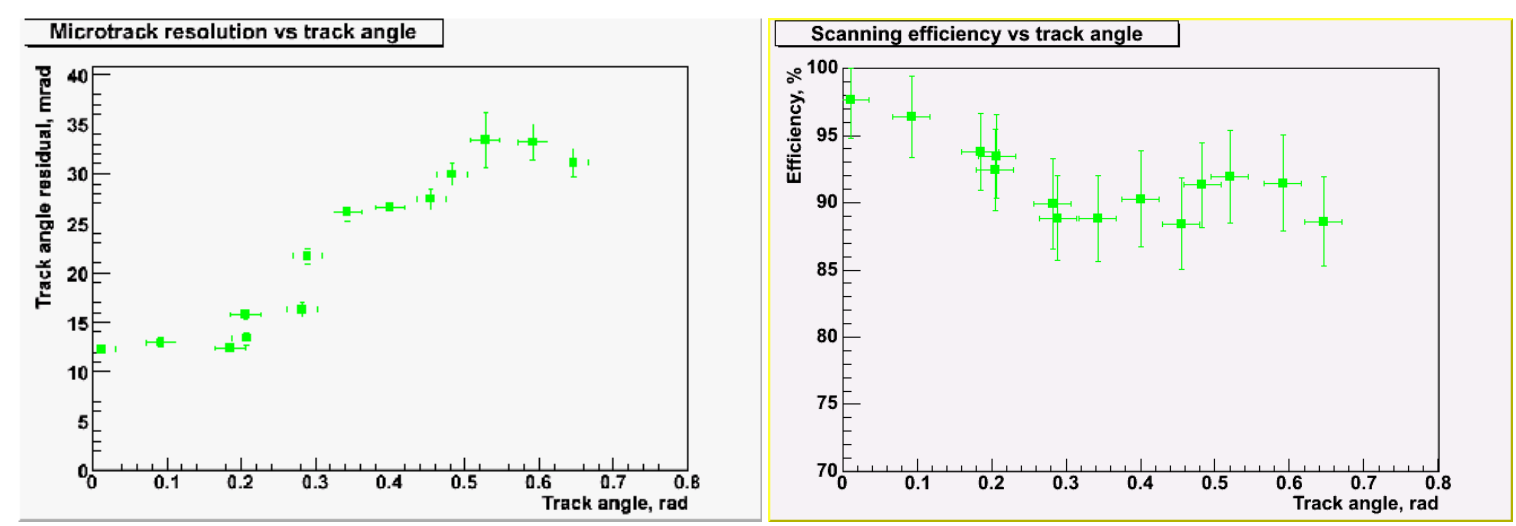

Figure 12. Dependence of the angular accuracy (left) and track reconstruction efficiency (right) vs track angle for a dry objective. 


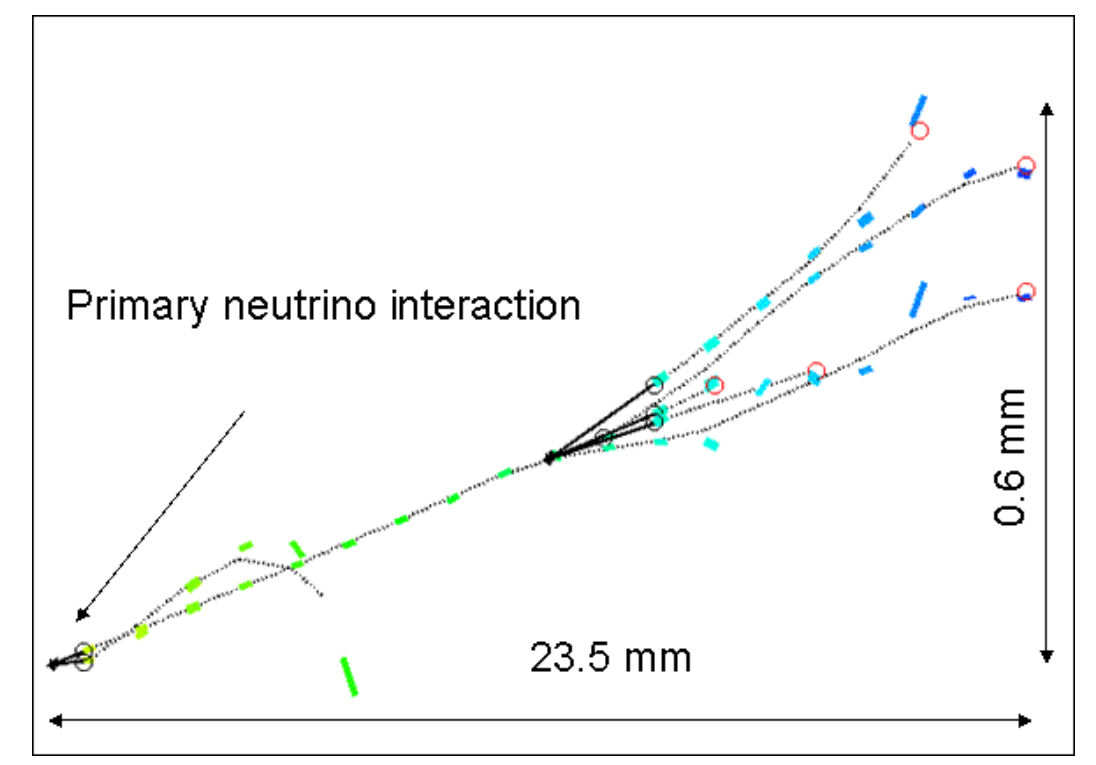

Figure 13. Detected, scanned and reconstructed muonless neutrino interaction in OPERA emulsion target by using dry objective lenses. Colored segments represent tracks in the emulsions. Black dotted lines indicate tracks fitted by a Kalman fitter algorithm. Continuous black lines indicate track matching to their common vertex.

\section{Acknowledgments}

We warmly thank all the colleagues of the OPERA collaboration and acknowledge support from our funding agencies.

\section{References}

[1] C.F. Powell et al., The study of elementary particles by the photographic method, Pergamon Press, New York (1959).

[2] W.H. Barkas, Nuclear research emulsion, Academic Press, London (1963).

[3] J. Nishimura, Soryusiron Kenkyu, Japan 12 (1956) 24.

[4] C.M.G. Lattes et al., Chacaltaya emulsion chamber experiment, Proc. of the International Cosmic Ray Conference, Jaipur 5 (1963) 326.

[5] S.L.C. Barroso et al., New clean example of Centauro species, observed by Chacaltaya Emulsion Chamber Experiment, Proc. of the XXV International Cosmic Ray Conference, Durban, (1997).

[6] S.G. Bayburina et al., Contribution to 24th International Cosmic Ray Conference by Chacaltaya and Pamir emulsion chamber experiments, Proc. of the XXIV International Cosmic Ray Conference, Rome 1995.

[7] M. Akashi et al., Large scale emulsion chamber experiment at Mt. Fuji, Proc. of Cosmic Rays and Particle Physics Conf., Newark, 1978

[8] J.Ren et al., Properties of hadron families observed with thick-type emulsion chambers at Mts. Kanbala and Fuji and search for Centauro events, Phys. Rev. D 38 (1998) 1417. 
[9] K. Niu, Discoveries of naked charm particle, and of lifetime difference among charm species carried out using compact ECC (Emulsion Cloud Chamber), I Intl. Workshop on Nuclear Emulsion, Nagoya, 12-14 June 1998.

[10] K. Niu et al., A possible decay in flight of a new type particle, Progr. Theor. Phys. 46 (1971) 1644.

[11] K. Hoshino et al., On the x Particles Observed in the Cosmic Ray Jet Showers, Contr. Paper at the XIV International Cosmic Ray Conference, Munich, 7 (1975) 2442.

[12] K. Hoshino et al., X-particle production in $205 \mathrm{GeV/c}$ proton interactions, Progr. Theor. Phys. 53 (1975) 1859.

[13] N. Ushida et al., Observation of a pair decay of short-lived neutral particles produced in $400 \mathrm{GeV/c}$ proton interactions, Lett. Nuovo Cim. 23 (1978) 577.

[14] H. Fuchi et al., Direct observation of charmed particle pairs produced in $340 \mathrm{GeV/c}$ negative pion interactions in an emulsion chamber, Lett. Nuovo Cim. 31 (1981) 199.

[15] N. Ushida et al., Experimental details on lifetime measurements of neutrino produced charmed particles in a tagged emulsion spectrometer, Nucl. Instrum. Meth. A 224 (1984) 50.

[16] E. Eskut et al., Final results on $v_{\mu}$ to $v_{\tau}$ oscillation from the CHORUS experiment, Nucl. Phys. B 793 (2008) 326.

[17] K. Kodama et al., Observation of tau neutrino interactions, Phys. Lett. B 504 (2001) 218.

[18] OPERA collaboration, Experiment Proposal. OPERA: An appearance experiment to search for numu-nutau oscillations in the CNGS beam, CERN/SPSC 2000-028, SPSC/P318, LNGS P25/2000, http://operaweb.web.cern.ch/operaweb/documents/index.shtml.

[19] OPERA collaboration, R. Acquafredda et al., First events from the CNGS neutrino beam detected in the OPERA experiment, New J. Phys. 8 (2006) 303 hep-ex/0611023.

[20] S. Aoki et al., The fully automated emulsion analysis system, Nucl. Instrum. Meth. B 51 (1990) 466.

[21] T. Nakano et al., Automatic analysis of nuclear emulsion, Ph.D. Thesis, University of Nagoya.

[22] G.Rosa et al., Automatic analysis of digitized TV images by a computer driven optical microscope, Nucl. Instrum. Meth. A 394 (1997) 357.

[23] N.Armenise et al., High-speed particle tracking in nuclear emulsion by last generation automatic microscopes, Nucl. Instrum. Meth. A 551 (2005) 261.

[24] N.Armenise et al., Hardware performace of a scanning system designed for high speed analysis of nuclear emulsions, Nucl. Instrum. Meth. A 568 (2006) 578.

[25] M.W. Davidson and M. Abramowitz, Optical microscopy, Olympus America Inc., New York (2003).

[26] http://micro.magnet.fsu.edu/primer/anatomy/immersion.html.

[27] I. Kreslo et al., A novel automatic film changer for high-speed analysis of nuclear emulsions, Nucl. Instrum. Meth. A 566 (2006) 327. 